\title{
Maternal exposure of mice to sodium $p$-perfluorous nonenoxybenzene sulfonate causes endocrine disruption in both dams and offspring
}

\author{
Caiyun Wang ${ }^{1)}$, Cuiyuan Jin ${ }^{1)}$, Wenqing $\mathrm{Tu}^{2)}$ and Yuanxiang $\mathrm{Jin}^{1)}$ \\ 1) Department of Biotechnology, College of Biotechnology and Bioengineering, Zhejiang University of Technology, Hangzhou 310032, \\ China \\ 2) Research Institute of Poyang Lake, Jiangxi Academy of Sciences, Nanchang 330029, China
}

\begin{abstract}
The toxicity of certain novel perfluoroalkyl substances (PFCs) has attracted increasing attention. However, the toxic effects of sodium $p$-perfluorous nonenoxybenzene sulfonate (OBS) on the endocrine system have not been elucidated. In this study, OBS was added to the drinking water during the pregnancy and lactation of the healthy female mice at dietary levels of $0.0 \mathrm{mg} / \mathrm{L}(\mathrm{CON}), 0.5 \mathrm{mg} / \mathrm{L}(\mathrm{OBS}-\mathrm{L})$, and $5.0 \mathrm{mg} / \mathrm{L}$ (OBS-H). OBS exposure during the pregnancy and lactation resulted in the presence of OBS residues in the placenta and fetus. We also analyzed physiological and biochemical parameters and gene expression levels in mice of the F0 and F1 generations after maternal OBS exposure. The total serum cholesterol (TC) and low-density lipoprotein cholesterol (LDL-C) levels were significantly increased in female mice of the F0 generation. The androgen levels in the serum and the ovarian mRNA levels of androgen receptor $(A R)$ also tended to increase after maternal OBS exposure in the F0 generation mice. Moreover, maternal OBS exposure altered the mRNA expression of endocrine-related genes in male mice of F1 generation. Notably, the serum TC and LDL-C levels were significantly increased in 8-weeks-old male mice of the F1 generation, and the serum high-density lipoprotein cholesterol (HDL-C) levels were decreased in 24-week-old male mice of the F1 generation. These results indicated that maternal OBS exposure can interfere with endocrine homeostasis in the F0 and F1 generations. Therefore, exposure to OBS during pregnancy and lactation has the potential toxic effects on the dams and male offspring, which cannot be overlooked.
\end{abstract}

Key words: Sodium p-perfluorous nonenoxybenzene sulfonate (OBS), Mice, Maternal exposure, Endocrine, F1 generation

SINCE THE 1950s, perfluoroalkyl substances (PFCs) have been used in various fields due to their excellent characteristics [1,2]. At present, a host of studies has revealed that PFCs have multiple toxic effects on animals [3-7]. Therefore, some commercial alternatives of PFCs are emerging [8]. Sodium $p$-perfluorous nonenoxybenzene sulfonate (OBS) is a substitute for PFCs with an aromatic moiety, the strong carbon fluorine (C-F) bonds provide for high thermal, chemical, and biological stability of OBS. OBS is cost-effectiveness and is thus widely used in China and some other countries. Statistical data indicated that total annual production capacity of the domestic OBS manufacturers has reached nearly 3,500 tons [9]. OBS was reported to have acute toxicity similar

Submitted Dec. 9, 2020; Accepted Apr. 8, 2021 as EJ20-0781

Released online in J-STAGE as advance publication May 11, 2021 Correspondence to: Yuanxiang Jin, Department of Biotechnology, College of Biotechnology and Bioengineering, Zhejiang University of Technology, 18, Chaowang Road, Hangzhou 310032, People' Republic of China.

E-mail: jinyx@zjut.edu.cn to that of perfluorooctane sulfonate (PFOS) according to the Globally Harmonized System criteria [10, 11]. In addition, the PBT (persistent, bioaccumulative and toxic properties) characteristics of OBS have not been improved compared with those of PFOS and perfluorooctanoic acid (PFOA) [12]. Potential pollution by OBS has been detected in surface water near the oil-field areas of North China [13]. A recent study also detected OBS in human blood [14], indicating that the potential health risk of OBS cannot be ignored.

Maintenance of endocrine function is regulated by the complete and healthy endocrine system. The endocrine system includes a group of specialized organs or glands that secrete hormones directly into the circulation to maintain growth, metabolism, and homeostasis of the organism [15]. Many environmental chemicals disrupt the animal endocrine system [16-20]. These chemicals interfere with the endocrine system by various routes involved in steroid receptor binding (agonists), blockade of steroid receptor binding (antagonists), or disruption of steroid biosynthesis [21-23]. Recently, some PFCs have 
been identified as endocrine disruptors in animals [24]. For example, PFCs can interfere with the functions of steroid hormone receptors [25], act as endocrinedisrupting chemicals [26], and disrupt endocrine signaling $[27,28]$. However, the endocrine toxicity of OBS is unknown. Some PFCs were reported to selectively cross the placenta [29]. Generally, developing organisms are more vulnerable and sensitive to environmental chemicals or stressors during embryonic period [30, 31]. Therefore, the exposure to PFCs during the sensitive period is also becoming a great concern [32].

According to the previous studies, OBS disturbs the balance of hepatic metabolism and the gut microbiota composition in zebrafish $[33,34]$, causes liver vacuolation, and induces a dysfunction of the gut barrier in mice [35]. Additionally, OBS should be a Category III chemical due to acute toxicity because the LC50 (96 h) value of OBS in zebrafish is $25.5 \mathrm{mg} / \mathrm{L}$ [13]. The acute oral LD50 value of PFOS in male C57BL/6J mice was reported to be $0.579 \mathrm{~g} / \mathrm{kg}$ body weight (BW) [36]. However, these studies were focused on the toxic effects of OBS on exposed entities, and it is unclear whether early offspring can be exposed to OBS through maternal contact. In the present study, C57BL/6 female mice were exposed to various concentrations of OBS $(0.0,0.5$, and $5.0 \mathrm{mg} / \mathrm{L}$ ) in drinking water during the gestation and lactation periods in the F0 generation. The F1 generation was fed a standard diet after weaning. Subsequently, we analyzed whether OBS can be transferred to F1 generation mice through the placental barrier. We also measured the weight of ovaries and testes, the levels of the main hormones, and transcription levels of the genes relevant to sex hormone receptors, cholesterol transport, and steroidogenesis in the dams and offspring (Fig. 1). This study investigated endocrine-disrupting effect of maternal exposure to OBS on the F0 and F1 generations.

\section{Materials and Methods}

\section{Chemicals, experimental animals and studies}

The OBS (purity of 98\%) was provided by Ningbo Yongshen Trading Co., Ltd. (Zhejiang, China). During the experimental period, OBS was dissolved in acetic acid, and the volume (acetic acid): the volume (drinking water) $=1: 20,000$ in every group.

All mouse experiments abided by Guiding Principles of Zhejiang University of Technology. The animals were treated humanely and every effort was made to minimize animal suffering. We purchased the 8-week-old C57BL/6 female $(n=30)$ and 10 -week-old C57BL/6 male mice $(n=30)$ from the China National Laboratory Animal Resource Center (Shanghai, China). All mice were housed in animal facilities for 1 week prior to experimentation with a 12 hours light cycle before the beginning of experiments. During experimental period, OBS was administered through the drinking water to female mice at dietary levels of $0.0 \mathrm{mg} / \mathrm{L}(\mathrm{CON}), 0.5 \mathrm{mg} / \mathrm{L}$ (OBS-L) and $5.0 \mathrm{mg} / \mathrm{L}(\mathrm{OBS}-\mathrm{H})$, food and water were available ad libitum.

After 1 week, we randomly divided the female animals (F0, $n=30)$ into three groups (10 mice each) with similar body weights. Females were paired with males for fertilization. After detection of the vaginal plug in females, the males were removed from the cage, and females were exposed to a specific concentration of OBS until weaning of F1 generation mice. During the experiment, two 10-day-pregnant female mice were randomly selected from each group to detect whether maternal exposure resulted in OBS entry into the embryo through the placental barrier. The offspring (F1) were weaned at 3 weeks of age. Then, the animals were fed a standard diet and water. We divided the three groups of weaned mice into two groups (including a control group and two

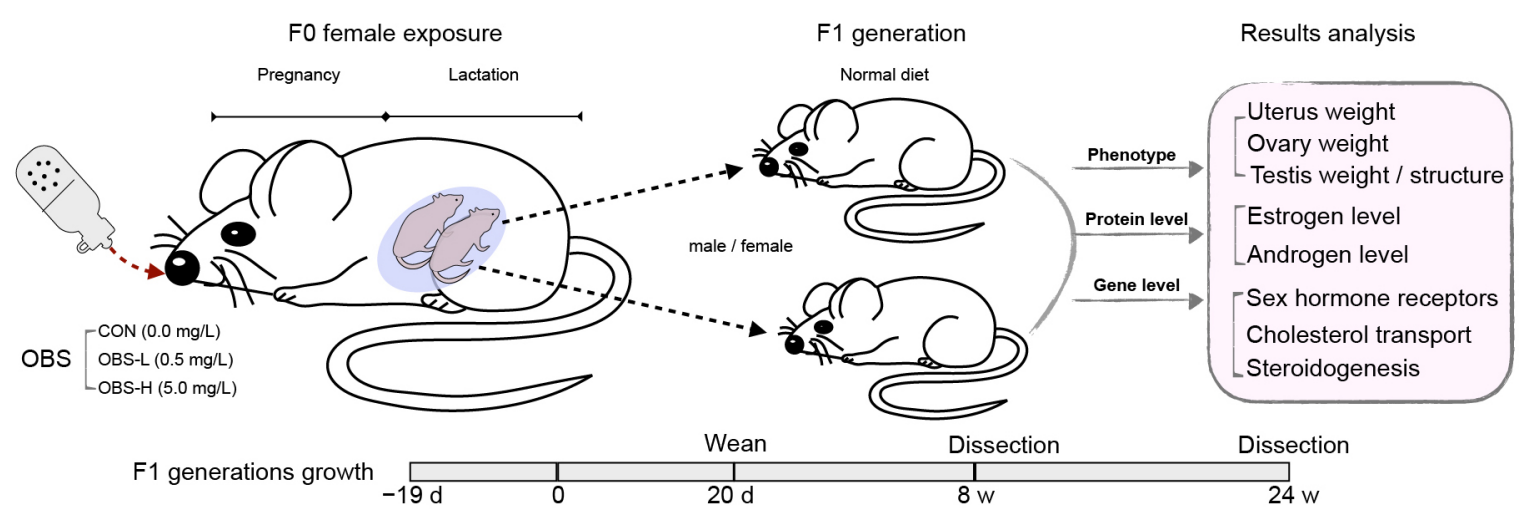

Fig. 1 Study design: Female adult C57BL/6 mice were fed a normal diet. During the gestation and lactation periods (marked as F0), OBS was added to the drinking water at dietary levels of 0 (CON), $0.5 \mathrm{mg} / \mathrm{L}$ (OBS-L), and $5.0 \mathrm{mg} / \mathrm{L}$ (OBS-H). The offspring (marked as F1) were weaned at 3 weeks of ages and then feed a standard diet and water then. Subsequently, the F0 female and F1 male animals $(n=8$ /group) were separately sacrificed for analysis. 
experimental groups of 8 mice each) and housed the animals in cages containing no more than three mice. Subsequently, a group of F1 generation mice was housed to 8 weeks of ages, and another group was housed to 24 weeks of ages. After weaning of F1 generation mice, the F0 mice ( $n=8 /$ group) were sacrificed and examined. The F1 generation ( $n=8$ /group) was sacrificed at 8 and 24 weeks of age (F1-8w and F1-24w, respectively). All blood samples were collected retro-orbitally as reported previously [37], and the testes, ovaries and uterus were rapidly removed and weighed. All tissues were rapidly frozen in liquid nitrogen and stored at $-80^{\circ} \mathrm{C}$ until use.

\section{Quantification of $\mathrm{OBS}$}

To detect the concentration of OBS from the testis (male) and uterus (female), the sample was spiked with an internal standard (potassium L-PFOS; $10 \mathrm{ng}$ ) first, and then ultrasonically extracted with $500 \mu \mathrm{L}$ of acetonitrile for 5 minutes. To remove lipids, we selected approximately 450 ul of extract to pass through an Oasis PRiME HLB column (1 cc/30 mg, Waters, MA). After processing the collected samples, we performed ultra performance liquid chromatography-tandem mass spectrometry (UPLC-MS/MS). We used an Agilent 1290 Infinity Series and Triple Quadruple 6420 from Agilent Technology (Santa Clara, CA, USA) to quantitative analysis OBS. Quantification was performed by the internal standard approach. And the average recovery rate of OBS was between $95.8 \%$ and $116.0 \%$ for tissues.

\section{Histopathological analysis of the testis}

A testicular, randomly selected 3 mice from F1 generation 8 male mice in each group, was fixed directly in $4 \%$ formaldehyde or Bouin's fixative for pathological observation. The samples were embedded in paraffin and cut into $5 \mu \mathrm{m}$ thick, stained with hematoxylin and eosin (H\&E). According to previous studies, we analyzed the spermatogenesis in the testicular sections [38-41].

Immunohistochemical analysis in the testis- The testis of three mice from each group were randomly selected for immunohistochemical analysis, and paraformaldehyde-fixed testis tissues were cut into 4$\mu \mathrm{m}$-thick sections and embedded in paraffin. After deparaffination with xylene and hydration through a graded ethanol series, the slices were incubated with a peroxidase-blocking solution for $10 \mathrm{~min}$. Antigen retrieval was performed according to the manufacturer's instructions. The optimal effect dilution for the polyclonal ER $\alpha$ (GB13205, Servicebio) and 17ß-HSD (Bs-3905R, Bioss) antibody was determined to be 1:200 and 1:300. Place the slices flat in a wet box and incubate overnight at $4^{\circ} \mathrm{C}$. Then, incubate the slices with the secondary antibody $(1: 200)$ of the corresponding species for
$50 \mathrm{~min}$ at room temperature. The slices were developed by DAB color liquid. Finally, sections were counterstained with hematoxylin and Alcian blue, dehydrated and mounted for microscope observation [42].

\section{Determination of serum levels of hormone, $T C$, $H D L-C$, and $L D L-C$ in mice}

All reagents were prepared before starting assay procedure. The serum TC, HDL-C and LDL-C were detected according to the manufacturer's instructions. Then, the level of estrogen (CK-E20462M, Biosamite) and androgens (CK-E92576M, Biosamite) in the serum of each mouse were detected using commercial enzymelinked immunosorbent assay (ELISA) kits (Jijin Chemical Technology Co., Ltd., Shanghai, China) according to the corresponding instructions. The optical density (O.D.) at $450 \mathrm{~nm}$ was measured on a microplate reader (Power wave XS, Bio-TEK, USA) by using the specific 96-well microplate. Among them, the standard curve was generated by plotting the average O.D. (450 nm) obtained for each of the six standard concentrations. The standard curve was used to determine the hormone levels in an unknown sample.

\section{Western blotting analysis}

Testicular tissues were washed with PBS, lysed with RIPA lysis buffer (Merck, USA). Protein concentrations were quantified using BCA Kits according to the manuscript's instructions. Tissue lysates were run on SDS/PAGE and transferred to PVDF membranes (Millipore, USA). Before immunoblotting, PVDF membrane were blocked with TBS-T containing non-fat milk (5\%) for $1 \mathrm{~h}$ and then blotted with an antibody against $\mathrm{ER} \alpha$ (GB111843, Servicebio) and 17 $\beta$-HSD (Bs-3905R, Bioss) overnight at $4^{\circ} \mathrm{C}$. Among, the optimal effect dilution for the antibodies ER $\alpha$ and 17 $\beta$-HSD in western blotting was determined to be 1:500 according to the corresponding instructions. After extensive washing with TBS-T, membranes were further incubated with horseradish peroxidase (HRP)-labeled secondary antibody $(1: 10,000)$ for $1 \mathrm{~h}$ at room temperature. Finally, membranes were incubated with ECL detection kits and exposed with Luminescent Imaging Workstation (Tanon, China). The relative protein content was quantified by ImageJ.

\section{Total RNA extraction and gene expression analysis}

Total RNA was harvested from tissues (testis, ovary) in BioZOL reagent. The concentration of total RNA was measured by absorbance at $260 \mathrm{~nm}$ using an ultravioletvisible spectrophotometer (Bio-TEK, USA). The cDNA preparation from $500 \mathrm{ng}$ of RNA was performed using qScript first-strand cDNA synthesis kit (Vazyme, China). 
RT-qPCR was performed using SYBR Green (Vazyme, China) on an Eppendorf MasterCycler ep RealPlex2 thermocycler machine (Wesseling-Berzdorf, Germany). The quantification of relative gene expression was accomplished as previously described [43, 44]. The sequences of the primers of genes related to estrogen and androgen biosynthesis, steroidogenesis, cholesterol transport in mice were used according to previous reports [41, 45] and the levels of 18s RNA (Forward: 5'-CGAACGT CTGCCCTATCAACTT-3'; Reverse: 5'-CCGGAATCGA ACCCTGATT-3') transcripts were determined, which served as a housekeeping gene [46].

\section{Statistical analysis}

Data were analyzed using GraphPad Prism version 7 (GraphPad Software) and SPSS 13.0 (SPSS, Chicago, Illinois) between the control group and the treated group by one-way ANOVA followed by Dunnett's test, twoway ANOVA followed by Tukey's test, and KruskalWallis test. They were evaluated as the mean \pm standard error of the mean (SEM). The results were considered significantly changed when $p$-value $\leq .05$.

\section{Results}

\section{Bioaccumulation of $\mathrm{OBS}$ in the embryo, placenta, uterus, and testes}

As shown in Fig. 2A and B, the presence of OBS was detected in the embryo and placenta in a dose-dependent manner. Subsequently, we assayed OBS in the uterus of the F0 generation and in the testes (male) and uterus (female) of the F1 generation after maternal OBS exposure (Fig. 2C). The results showed that OBS was detected in maternal uterus after maternal OBS exposure. However, little or no OBS was found in the F1-8w and F1-24w mice (no differences from the control group).

\section{Effect of maternal OBS exposure on body weights and the weight of uterus, ovaries, testes and epididymal fat}

We counted the weight of F1 generation mice and found that during the growth from birth to 24 weeks of age, only 8 weeks old and 18 weeks old mice had temporary change of body weights in OBS-H group, and there was no significant statistical difference between the body
A
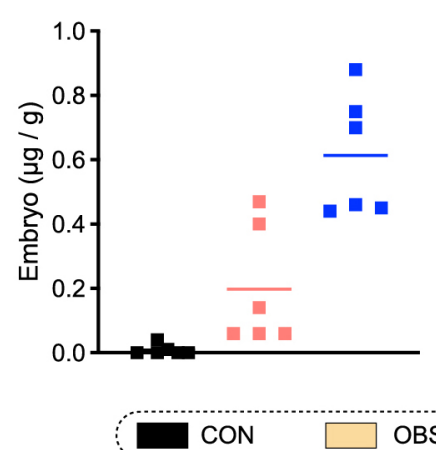

B

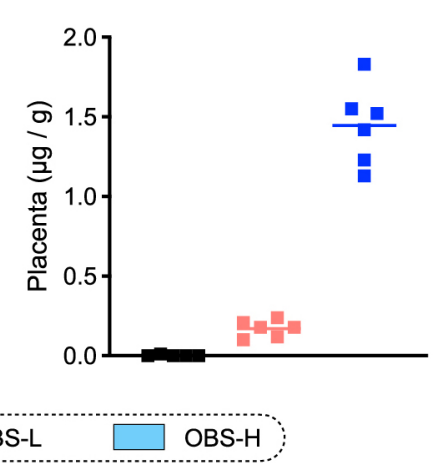

$\mathrm{C}$

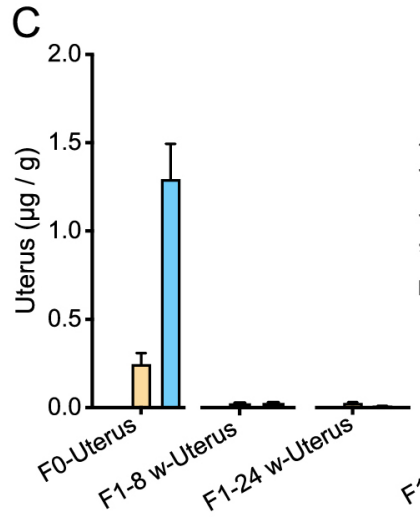

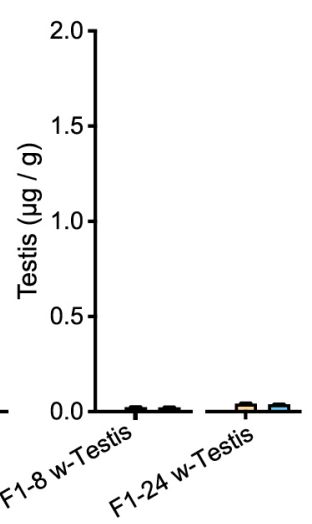

$\mathrm{F}$

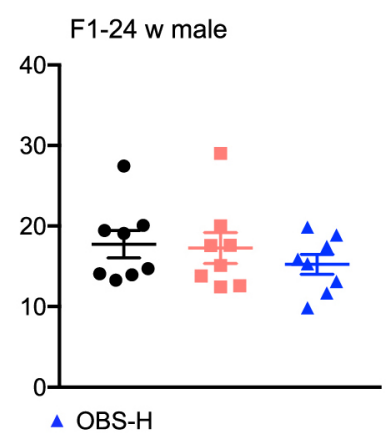

$\mathrm{D} \rightarrow \mathrm{F} 1-\mathrm{CON} \rightarrow \mathrm{F} 1$-male-CON $\rightarrow$ F1-female-CON

-F1-OBS-L F1-male-OBS-L $-\mathrm{F} 1$-female-OBS-L

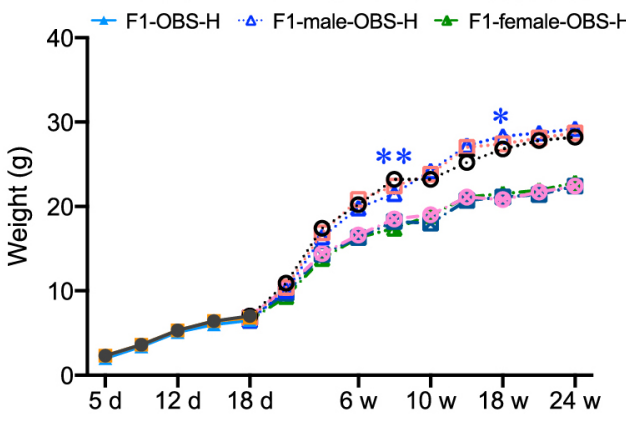

$\mathrm{E}$

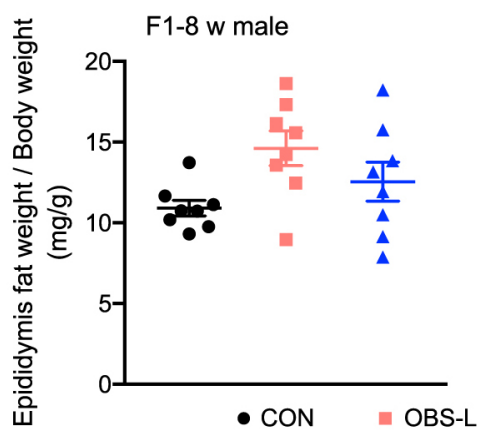

Fig. 2 Maternal exposure to OBS resulted in the bioaccumulation of OBS and the effects of OBS on the body weight and relative fat weight of the F1 generation. (A-B) Bioaccumulation of OBS in the embryo and placenta; (C) bioaccumulation of OBS in the uterus and testis of the F0 and F1 generations; (D) body weight gain in the offspring; (E-F) relative fat weight of F1-8w and F1-24w male mice. The values are presented as the mean $\pm \operatorname{SEM}(n=8$ /group). The blue asterisk indicated the difference between OBS-H and the control group of the F1 generation male mice. ${ }^{*} p \leq 0.05, * * p \leq 0.01$ versus the control. 
weights of the treatment group and the control at other days or weeks of age after maternal OBS exposure. However, under the same treatment group conditions, whether in control group or treatment group, as the week age of the mice increases, the weight of the mice has changed significantly (Fig. 2D and Table S1), which showed the influence of time on the weights of the mice more obvious. Additionally, we also determined the weight of the uterus, ovary, testes, and epididymal fat in F0 and F1 generation mice. Relative weight of the uterus, ovaries, and epididymal fat was not statistically significantly different between the control and OBStreated groups in F0 and F1 female mice (Table 1,

Table 1 Effects of maternal OBS exposure on the weight of ovaries and uterus of the dams and offspring

\begin{tabular}{lccc}
\hline \multirow{2}{*}{$\begin{array}{c}\text { Tissue/body } \\
\text { weight }(\mathrm{mg} / \mathrm{g})\end{array}$} & \multicolumn{3}{c}{ Group } \\
\cline { 2 - 4 } $\begin{array}{c}\text { F0 female } \\
\text { Ovary }\end{array}$ & $0.624 \pm 0.036$ & $0.706 \pm 0.031$ & $0.627 \pm 0.035$ \\
Uterus & $4.116 \pm 0.257$ & $4.776 \pm 0.304$ & $3.836 \pm 0.281$ \\
\hline F1-8w female & & & \\
Ovary & $0.561 \pm 0.024$ & $0.603 \pm 0.015$ & $0.505 \pm 0.084$ \\
Uterus & $2.736 \pm 0.321$ & $3.957 \pm 0.361$ & $3.376 \pm 0.710$ \\
F1-24w female & & & \\
Ovary & $0.533 \pm 0.037$ & $0.589 \pm 0.044$ & $0.422 \pm 0.043$ \\
Uterus & $3.437 \pm 0.370$ & $3.155 \pm 0.217$ & $3.128 \pm 0.200$ \\
\hline
\end{tabular}

The values are presented as the mean $\pm \operatorname{SEM}(n=8$ /group).
Fig. 2E and F). However, the OBS-H treatment group manifested a considerable increase in the relative weight of the testes in F1-8w male mice (Fig. 3A). In contrast, the relative weight of the testes was not changed in F1-24w male mice (Fig. 3B).

We then determined whether maternal OBS exposure directly resulted in histopathological changes in the testicles of the F1 generation. The results of the H\&E staining indicated a significant reduction in the number of spermatocytes and spermatozoa in the seminiferous tubules in the testes of F1-8w male mice after maternal OBS exposure (Fig. 3C). In contrast, the number of spermatocytes and spermatozoa in the testes of F1-24w male mice had no visible changes; however, we observed marginal condensation of the chromatin in the round spermatids (Fig. 3D). Additionally, the Kruskal-Wallis $\mathrm{H}$ test was used for H\&E staining results to compare the distribution of CWWS scores in different groups (Table S2). The results showed that the median CWWS scores of F1-8w and F1-24w mice in the CON group were 1.065 $(n=3)$ and $0.976(n=3)$, in the OBS-L group $0.672(n=$ $3)$ and $1.073(n=3), 0.746(n=3)$ and $1.242(n=3)$ in the OBS-H group, and the median of the total CWWS score is $0.780(n=9)$ and $1.066(n=9)$. Although the distribution of CWWS scores in each group was not all the same, the difference was not statistically significant $((\mathrm{H}=4.356, p=0.113)$ and $(\mathrm{H}=1.867, p=0.393))$.

\section{Effects on serum levels of TC, HDL-C, and LDL-C}

Cholesterol is an important substrate for the estrogen
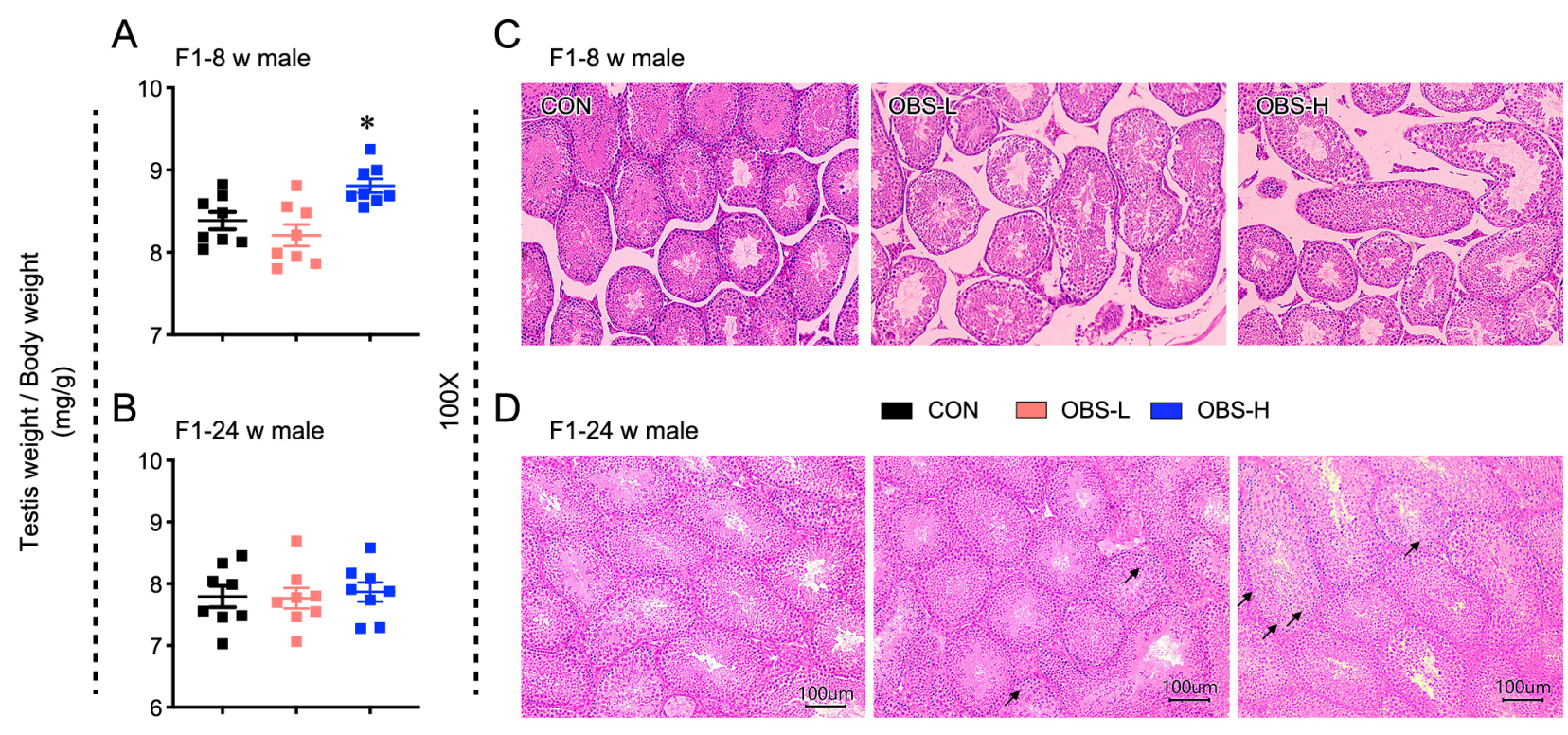

Fig. 3 Effects of maternal OBS exposure on the testis in the F1 generations. (A-B) The relative weights of the testis in the F1 generations; (C-D) morphological alterations in the H\&E stained sections of in the testes of the F1 generations. (The arrows represented the marginal condensation of chromatin in round spermatids). The values are presented as the means $\pm \operatorname{SEM}(n=8 /$ group). ${ }^{*} p \leq 0.05$ versus the control. 
and androgen synthesis. As shown in Table 2, the serum levels of TC, HDL-C, and LDL-C in the F0 and F1 generations were assayed. OBS exposure significantly increased the serum levels of TC and LDL-C in F0 generation mice; however, no statistically significant differences were observed in the serum levels of HDL-C. Similarly, the serum levels of TC, HDL-C, and LDL-C in F1-8w male mice presented a trend similar to that observed in the F0 generation. In contrast, the serum levels of TC and LDL-C in F1-24w male mice did not have distinct changes. However, a significant decrease was observed in the serum levels of HDL-C.

\section{Maternal OBS exposure altered the serum levels of estrogen and androgen, the testes protein levels of ER $\alpha$ and17ß-HSD}

To determine whether maternal OBS exposure changed the synthesis of estrogen and androgen in ovaries (F0 generation) and testes (F1 generation), we measured the serum levels of estrogen and androgen in mice. The level of androgen had an increasing trend, and the estrogen level was decreased in the F0 generation after maternal exposure to $5.0 \mathrm{mg} / \mathrm{L} \mathrm{OBS}$; however, the levels of these two hormones did not change in the OBS-L group (Fig. 4A). The androgen levels were significantly decreased in F1-8w males after maternal exposure to OBS compared with that in the control, and the estrogen levels did not change in the OBS-treated groups (Fig. 4B). Interestingly, the changes in the levels of these hormones in F1-24w male mice were different from those in F1-8w male mice. The estrogen levels were decreased in

Table 2 Effects of maternal OBS exposure on some biochemical indexes in the serum of the dams and offspring

\begin{tabular}{lcll}
\hline \multirow{2}{*}{ Index } & \multicolumn{3}{c}{ Group } \\
\cline { 2 - 4 } & CON & OBS-L & OBS-H \\
\hline F0 female & & \\
TC $(\mathrm{mmol} / \mathrm{L})$ & $3.32 \pm 0.17$ & $3.57 \pm 0.12$ & $4.03 \pm 0.20^{*}$ \\
HDL-C $(\mathrm{mmol} / \mathrm{L})$ & $2.38 \pm 0.13$ & $2.53 \pm 0.12$ & $2.29 \pm 0.11$ \\
LDL-C $(\mathrm{mmol} / \mathrm{L})$ & $0.42 \pm 0.04$ & $0.45 \pm 0.05$ & $0.62 \pm 0.05^{*}$ \\
F1-8w male & & & \\
TC $(\mathrm{mmol} / \mathrm{L})$ & $3.95 \pm 0.11$ & $3.57 \pm 0.54$ & $4.68 \pm 0.21^{*}$ \\
HDL-C $(\mathrm{mmol} / \mathrm{L})$ & $1.26 \pm 0.04$ & $1.15 \pm 0.07$ & $1.14 \pm 0.04$ \\
LDL-C (mmol/L) & $0.16 \pm 0.01$ & $0.22 \pm 0.02$ & $0.26 \pm 0.02^{* *}$ \\
F1-24w male & & & \\
TC (mmol/L) & $3.37 \pm 0.22$ & $2.88 \pm 0.20$ & $3.06 \pm 0.12$ \\
HDL-C (mmol/L) & $2.54 \pm 0.24$ & $1.76 \pm 0.06^{* *}$ & $1.73 \pm 0.06^{* *}$ \\
LDL-C (mmol/L) & $0.69 \pm 0.07$ & $0.75 \pm 0.09$ & $0.77 \pm 0.09$ \\
\hline
\end{tabular}

The values are presented as the mean $\pm \operatorname{SEM}(n=8$ /group). Asterisks indicates significant differences between the control and OBS-treated groups $(* p \leq 0.05, * * p \leq 0.01)$. the $5.0 \mathrm{mg} / \mathrm{L}$ OBS group, and the androgen levels were not different with those in the control in F1-24w males after maternal exposure to OBS (Fig. 4C).

Subsequently, we determined testes protein levels of ER $\alpha$ and $17 \beta-H S D$ of the F1 generation by immunohistochemical and western blotting analysis. The results indicated a significant reduction in protein levels of ER $\alpha$ and $17 \beta$-HSD of the testes of F1-8w male mice after maternal OBS exposure (Fig. 4D and F, Fig. S1A). Additionally, protein levels of ER $\alpha$ of the testes of F1-24w male mice had no significant changes and 17ß-HSD had a significant increase. (Fig. 4E and G, Fig. S1B).

\section{Maternal OBS exposure altered the expression of the genes associated with sex hormone receptors and cholesterol synthesis}

The result of real-time qPCR (Fig. 5A) indicated that maternal OBS-H exposure caused an increase in the levels of various genes, including estrogen receptors (ER $\alpha$ and ER $\beta$ ) and cholesterol synthesis-related HMGCoA reductase, but did not significantly affect the expression of the androgen receptors (AR) or cholesterol synthesis-related HMG-CoA synthase in the F0 generation. However, the levels of these genes were profoundly decreased after maternal OBS-H exposure in the F1-8w male mice (Fig. 5B). In contrast, the expression of the genes of ER $\beta$ and HMG-CoA synthase was significantly increased in the OBS-H group in F1-24w male mice, and the levels of the genes of AR, ER $\alpha$, and HMG-CoA reductase were not changed compared with those in the control (Fig. 5C).

\section{Maternal OBS exposure altered the expression of the genes associated with cholesterol transport and steroidogenesis}

Steroid hormone synthesis starts from cholesterol. Therefore, we detected the effect of maternal OBS exposure on the transcription of the key genes involved in cholesterol transport and steroidogenesis in the mice of the F0 and F1 generations. Maternal OBS exposure in the OBS-H groups considerably increased the mRNA levels of scavenger receptor class B type 1 (SR-B1), peripheral benzodiazepine receptor (PBR), and cytochrome p450 side-chain cleavage enzyme (P450SCC) in the F0 generation compared with those in the control. Except for 17- $\beta$-hydroxysteroid dehydrogenase (17 $\beta$ HSD), the expression of other genes showed an increasing trend (Fig. 6A). Notably, the expression of these genes was not significantly altered in the OBS-L group. These results indicated that the effect of OBS was not concentration-dependent. In contrast, the mRNA levels of steroidogenic acute regulatory protein (STA-R), P450SCC, 3- $\beta$-hydroxysteroid dehydrogenase (3 $\beta$-HSD), 

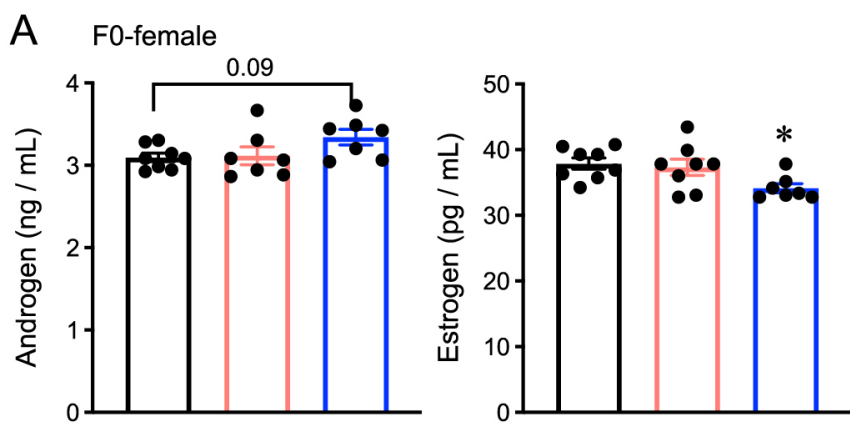

B F1-8 w male
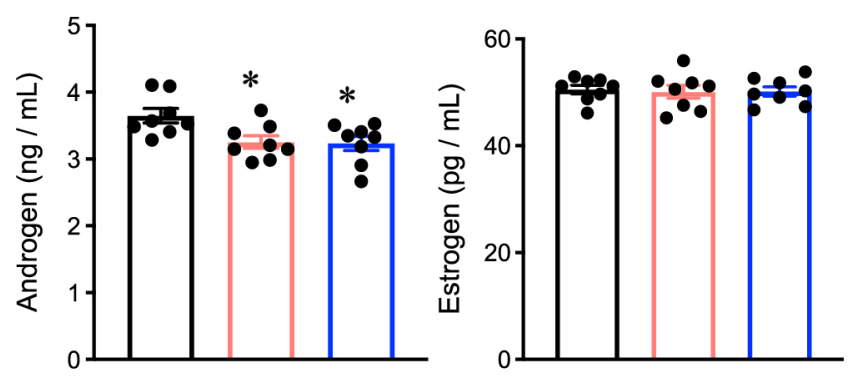

$$
\text { C F1-24 w-male }
$$

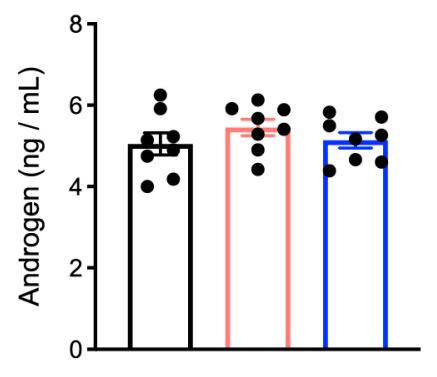

$\square$ CON $\square$ OBS-L

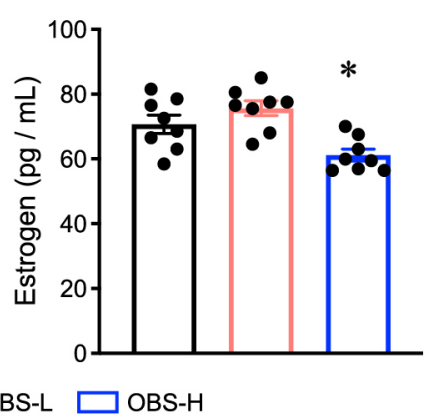

\section{F1-8 w male}

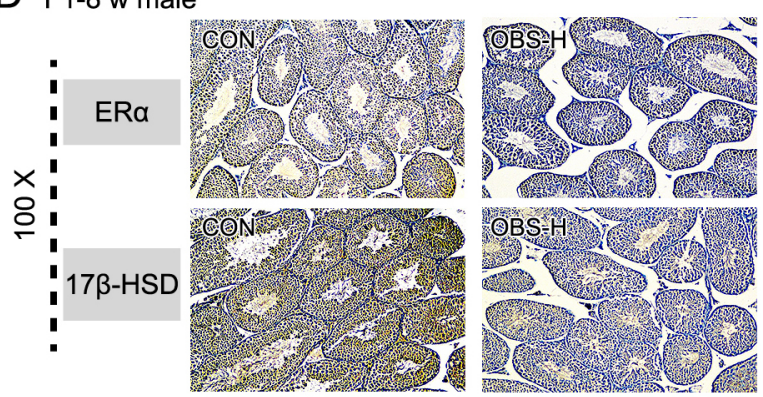

\section{E F1-24 w-male}

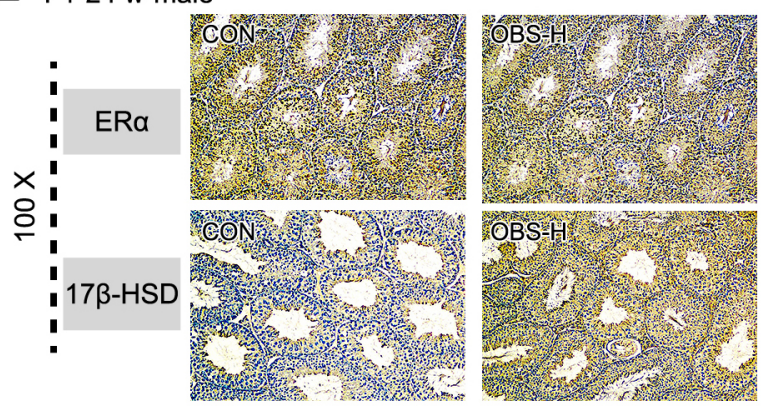

F F1-8 w male

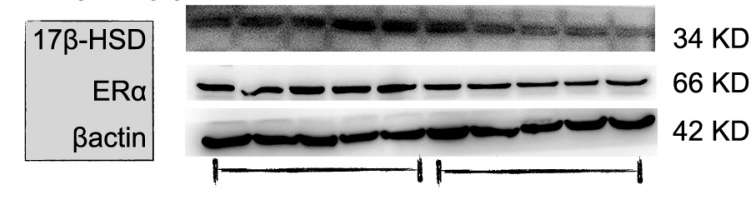

G F1-24 w male

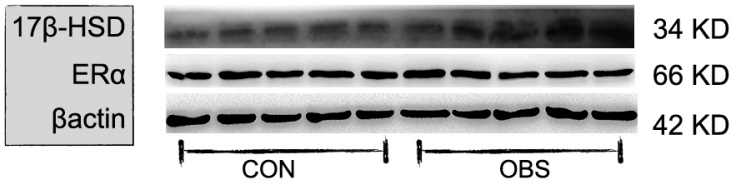

Fig. 4 Effects of maternal OBS exposure on the serum hormone levels. The results of ELISA of androgen and estrogen (A) in F0 female, (B) F1-8w and (C) F1-24w male mice; the immunohistochemistry result of ER $\alpha$ and 17ß-HSD (D) F1-8w and (E) F1-24w male mice; the protein expression of $\mathrm{ER} \alpha$ and $17 \beta-\mathrm{HSD}$ was measured by western blotting analysis (F) F1-8w and (G) F1-24w male mice. The values are presented as the mean $\pm \operatorname{SEM}\left(n=8\right.$ /group). ${ }^{*} p \leq 0.05$ versus the control.

and cytochrome P450 17 $\alpha$-hydroxysteroid dehydrogenase $(\mathrm{P} 45017 \alpha)$ were considerably decreased in F1-8w male mice of the OBS-H group compared with those in the control. Moreover, the levels of low-density lipoprotein receptor (LDL-R), SR-B1, PBR, and 17 $\beta$-HSD had a decreasing trend in the OBS-H group (Fig. 6B). However, except certain genes, the levels of most of the genes that were suppressed in F1-8w male mice were not significantly changed in the F1-24w male mice. For example, only the levels of LDL-R, 3 $\beta$-HSD, and 17 $\beta$-HSD were increased by maternal OBS-H treatment in the F1-24w- male mice (Fig. 6C).

\section{Discussion}

To date, a number of studies demonstrated that PFCs can act as endocrine disruptors [24, 47]. However, it is not known whether OBS has the endocrine disruptor effects in animals. In the present study, maternal OBS exposure might induce endocrine disruption in both the F0 and male F1 generations.

Undoubtedly, OBS can bioaccumulate in the animals (for example, in the serum, liver, gut, et c.), and a part of accumulated OBS can be excreted the feces [35]. In the present experiment, preliminary tests indicated exposure to OBS during pregnancy and lactation caused OBS bioaccumulation in the embryo and placenta (Fig. 2A, B and C). Thus, OBS, similar to other PFCs [48, 49], may pass through the placental barrier. The degradation of OBS requires approximately 400 days, and OBS is considered a highly recalcitrant pollutant [13]. Thus, short-term decomposition of OBS in the environment is difficult. In 

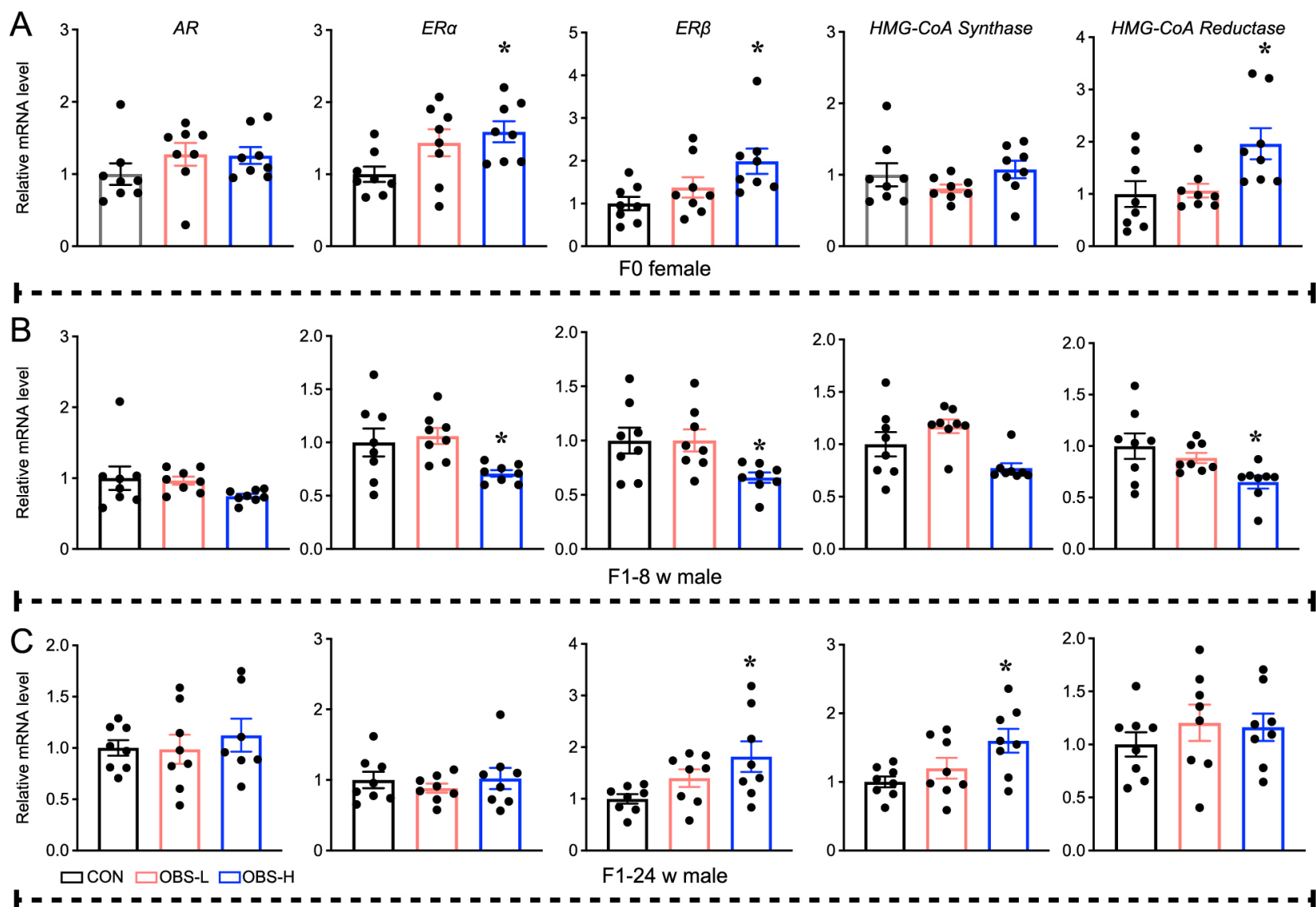

Fig. 5 Levels of transcription of mRNA of $A R, E R \alpha, E R \beta, H M G-C o A$ synthase, and $H M G-C o A$ reductase in the ovaries and testicles of the F0 and F1 generations. Gene expression levels represent the relative mRNA expression compared to that in the controls (A) in the ovaries of F0 female mice and (B) testes of F1-8w male mice and (C) F1-24w male mice. The values are presented as the means $\pm \operatorname{SEM}(n=8 /$ group $) . * p \leq 0.05$ versus the control.
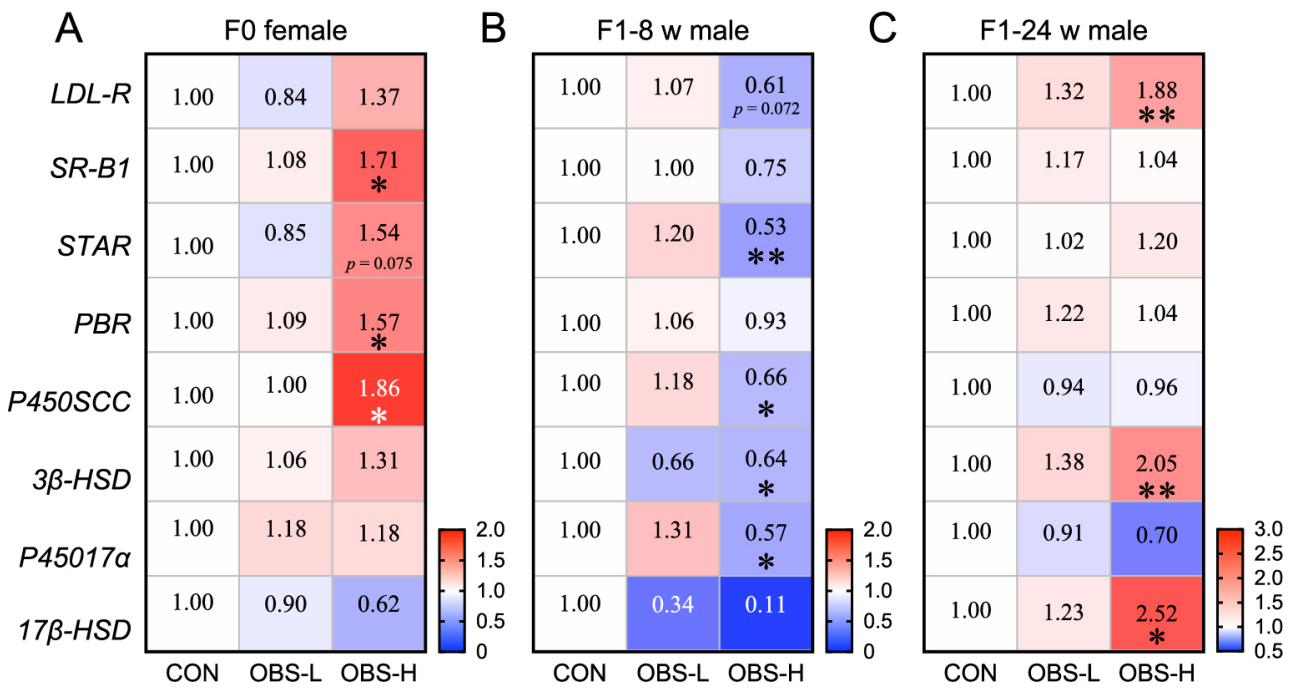

Fig. 6 Effects of maternal OBS exposure on the levels of the genes of cholesterol transport and steroidogenesis. The mRNA levels of cholesterol transport- and steroidogenesis-related genes (A) in the ovaries of F0 female and (B) testes of F1-8w male mice and (C) F1-24w male mice. The values are presented as the mean $\pm \operatorname{SEM}\left(n=8\right.$ /group). ${ }^{*} p \leq 0.05,{ }^{* *} p \leq 0.01$ versus the control. 
the present study, OBS remained in the maternal womb, however, the levels of OBS were very low in the testes and uterus of F1-8w and F1-24w mice (no differences from the control) (Fig. 2C). F1 generation mice were exposed to OBS early in life. The mice of F1 generation were normally raised after weaning; thus, we hypothesized that OBS entered the fetus was likely to be excreted by F1 generation mice during growth. This result is in agreement with our previous data on the detection of OBS in mouse feces [35].

Cholesterol is the base compound for the synthesis of all steroid hormones. Cholesterol is recognized and transported from the plasma into the cells by membrane bound LDL-R and SR-B1 [50, 51] and then participates in the synthesis of testosterone and estradiol. In the present study, serum TC and LDL-C levels were considerably increased in F0 generation mice after maternal OBS exposure (Table 2). Additionally, the expression of the genes of cholesterol synthesis (HMG-CoA reductase and HMG-CoA synthase) was significantly stimulated in the F0 generation. Cholesterol enters the cell and is transported through the mitochondrial membrane by STAR- and PBR-dependent transport that regulates cholesterol participation in the synthesis of steroid hormones $[23,50,52,53]$. The mRNA levels of the key genes involved in cholesterol transport and testosterone synthesis, including $3 \beta-\mathrm{HSD}, 17 \beta-\mathrm{HSD}, \mathrm{P} 450-\mathrm{SCC}$, and P450 $17 \alpha$, are also important $[32,54]$. The levels of these genes were also increased in the F0 generation, except for $17 \beta$-HSD (Fig. 6A). This increase in the expression apparently implied an elevation in the androgen level after maternal OBS exposure. Thus, a trend of an increase in the androgen level was also observed in the F0 generation. Therefore, we hypothesized that exposure to OBS may interfere with endocrine homeostasis in F0 female mice, and individuals who are preparing to become pregnant or are pregnant should avoid the contact with OBS as much as possible.

A number of in vivo and in vitro studies indicated that some of PFCs may disrupt endocrine homeostasis [25]. Some novel alternative compounds, for example, F-53B, induced developmental and reproductive toxicity and disrupted thyroid endocrine function [55-57]. Recently, it was reported that chronic/prepubertal exposure to some of PFCs impaired spermatogenesis and steroidogenesis in the testes of male mice, and induced the change of the histological features in the testis [58, 59]. Moreover, many studies showed that some of the common male reproductive disorders of adult life might originate in the fetal. Some of PFCs was the major environmental pollutants that could affect the development of male reproductive system if exposed during early life [60, 61]. Therefore, we investigate whether OBS exposure in early life disrupted the endocrine system in male mice during puberty (F1-8w mice) and adulthood (F1-24w mice). The testis is an endocrine organ important for the homeostasis of androgen and estrogen [50]. Our results of the $H \& E$ staining of the testes revealed a reduction in spermatogenesis in F1-8w male mice and marginal condensation of the chromatin in the round spermatids in F1-24w male mice. This proved that According to the previous studies $[23,58]$, this type of changes usually occurred if the weight of the testes remained constant or decreased after the exposure. However, the weight of the testes in F1-8w male mice after maternal OBS-H treatment was increased in our study. Spermatogenesis is a complex differentiation process that requires the secretion of testosterone (an androgen) to provide nutrition and protect the sperm microenvironment [62-64]. The androgen level in the serum of F1-8w male mice after OBS treatment showed an increasing trend; mice at 8 weeks of age are in a critical period of rapid development. These considerations may explain why the sperm number declined and the weight of the testes was increased in F1-8w male mice after maternal exposure to OBS-H. The serum estrogen level in F1-8w male mice was not significantly different between the OBS-treated and control group. The transcriptional levels of estrogen and androgen receptors in the testis were detected by the RT-qPCR, the results showed that the transcription of the genes of sex hormone receptors (AR, ER $\alpha$, and ER $\beta$ ) was downregulated (Fig. 5B), and the protein levels of ER $\alpha$ of the testes decreased (Fig. 4D and F), indicating that OBS affected sex hormones in F1 generation mice by disturbing testicular development and that the damage to the body is mediated by the protein and gene levels. The expression of the genes related to cholesterol transport and steroidogenesis in F1-24w male mice showed different changes compared to those in the F1-8w male mice (Fig. 6C), and the serum androgen level and the protein levels of ER $\alpha$ of the testes was not changed after maternal OBS exposure during the gestation and lactation period. However, the results of H\&E staining revealed marginal condensation of chromatin in the round spermatids. On the one hand, during the growth from 8 weeks to 24 weeks, certain body functions of mice were constantly recovering. On the other hand, as the individuals grew, the residue of OBS in the body was decreased; however, the negative effect on the offspring could not be eliminated, indicating that the effect on the cholesterol transport and steroidogenesis after maternal OBS exposure during the gestation and lactation period could not be fully reversed within a short period of time.

De novo synthesis of all steroid hormones is known to begin with the conversion of cholesterol. A study published by Shi et al. [50] reported that the genes related to 
cholesterol transport and steroidogenesis play an important role in the synthesis of estrogen and androgen. The transcriptional levels of these genes were significantly downregulated in F1-8w male mice (Fig. 6B). A reduction in the expression of the genes involved in cholesterol transport and steroidogenesis was reported to result in a corresponding reduction in testosterone synthesis and an impairment of spermatogenesis in the testis [65-67]. Additionally, our results revealed that the protein levels of $17 \beta$-HSD the testes in F1-8w male mice kept the same change with the gene levels after maternal OBS-H exposure (Fig. 4G). 17 $\beta$-HSD in the steroidogenic pathway is a key regulator of testosterone production $[41,50]$. Moreover, mice at 24 weeks of age in protein and genes levels of $17 \beta-H S D$ both had the significant up-regulation (Fig. 4E and G, Fig. 6C). Therefore, it was possible that OBS induced an imbalance in the endocrine homeostasis in male offspring by disturbing cholesterol transport and steroidogenesis after maternal OBS exposure that cannot be fully recovered within a short period of time.

Androgens secreted by the testis are the key regulatory elements of androgen cell signaling in male animals and the precursor hormones for estrogen biosynthesis in the ovaries and other gonadal tissues [25, 68]. Change in androgen may cause abnormalities in the body during pregnancy [41, 69], and consequently change the level of estrogen [70]. In general, these changes in the maternal organism are assumed to result in the symptoms of aromatase deficiency in the newborns [71]. Aromatase plays an important and indispensable role in the conversion of androgens to estrogens [72]. Some studies have shown that the aromatase deficiency results in a decreased in androgen conversion into estrogen and androgen accumulation in patients [73-75]. We did not determine the effect of OBS on the aromatase; however, our results indicated that the changes in the estrogen and androgen levels were clearly detected in the F1 male generation. Therefore, the effect of maternal OBS exposure on the endocrine function of the F1 male generation was demonstrated. However, it is unclear whether the endocrine effects of OBS inhibited aromatase.

In summary, the results of this study indicate that maternal OBS exposure during pregnancy and lactation resulted in the presence of OBS residues in the placenta and fetus. Subsequent growth of the offspring resulted in gradual excretion of OBS remaining in the fetus. Additionally, the genes involved in sex hormone receptors, cholesterol transport, and steroidogenesis were activated, and the serum TC and LDL-C levels were increased in the F0 generation. The androgen levels in the serum were significantly increased after maternal OBS exposure in the F0 generation, which apparently caused abnormalities in the organism during pregnancy. Therefore, the mRNA levels of the key genes related to sex hormone receptors, cholesterol transport, and steroidogenesis and the serum levels of TC, HDL-C, and LDL-C were significantly changed even in the $\mathrm{F} 1$ generation after maternal exposure to OBS, indicating that OBS may affect endocrine function in the F1 generation. Thus, the data of the present study are useful for health risk assessment; however, identification of a specific target of OBS in the endocrine system needs additional analysis.

\section{Conflicts of Interest}

The authors declare that they have no known competing financial interests.

\section{Funding}

This work was supported by Zhejiang Provincial Natural Science Foundation of China (No. LZ20B070002).

\section{References}

1. Kissa E (2001) Fluorinated Surfactants and Repellents (2nd). Marcel Dekker, New York.

2. Lindstrom AB, Strynar MJ, Libelo EL (2011) Polyfluorinated compounds: past, present, and future. Environ Sci Technol 45: 7954-7961.

3. Bjork JA, Lau C, Chang SC, Butenhoff JL, Wallace KB (2008) Perfluorooctane sulfonate-induced changes in fetal rat liver gene expression. Toxicology 251: 8-20.

4. Wang M, Chen J, Lin K, Chen Y, Hu W, et al. (2011) Chronic zebrafish PFOS exposure alters sex ratio and maternal related effects in F1 offspring. Environ Toxicol Chem 30: 2073-2080.

5. Coperchini F, Awwad O, Rotondi M, Santini F, Imbriani
M, et al. (2017) Thyroid disruption by perfluorooctane sulfonate (PFOS) and perfluorooctanoate (PFOA). $J$ Endocrinol Invest 40: 105-121.

6. Geng D, Musse AA, Wigh V, Carlsson C, Engwall M, et al. (2019) Effect of perfluorooctanesulfonic acid (PFOS) on the liver lipid metabolism of the developing chicken embryo. Ecotoxicol Environ Saf 170: 691-698.

7. Pan Z, Yuan X, Tu W, Fu Z, Jin Y (2019) Subchronic exposure of environmentally relevant concentrations of $\mathrm{F}-53 \mathrm{~B}$ in mice resulted in gut barrier dysfunction and colonic inflammation in a sex-independent manner. Environ Pollut 253: 268-277.

8. Zhou X, Wang J, Sheng N, Cui R, Deng Y, et al. (2018) 
Subchronic reproductive effects of 6: 2 chlorinated polyfluorinated ether sulfonate (6: 2 cl-PFAES), an alternative to PFOS, on adult male mice. J Hazard Mater 358: 256 264.

9. Bao Y, Qu Y, Huang J, Cagnetta G, Yua G, et al. (2017) First assessment on degradability of sodium p-perfluorous nonenoxybenzene sulfonate (OBS), a high volume alternative to perfluorooctane sulfonate in fire-fighting foams and oil production agents in China. RSC Adv 7: 46948 46957.

10. Wang S, Huang J, Yang Y, Hui Y, Ge Y, et al. (2013) First report of a Chinese PFOS alternative overlooked for 30 years: its toxicity, persistence, and presence in the environment. Environ Sci Technol 47: 10163-10170.

11. Wang W, Mi X, Shi H, Zhang X, Zhou Z, et al. (2019) Adsorption behaviour and mechanism of the PFOS substitute OBS (sodium p-perfluorous nonenoxybenzene sulfonate) on activated carbon. $R$ Soc Open Sci 6: 191069.

12. Hwang Jisu (2016) PBT Evaluation and Treatability Study of Sodium $\rho$-Perfluorinated Noneoxybenzen Sulfonate. Marcel Dekker, Tsinghua University (In Chinese).

13. Xu L, Shi Y, Li C, Song X, Qin Z, et al. (2017) Discovery of a novel polyfluoroalkyl benzenesulfonic acid around oilfields in northern China. Environ Sci Technol 51: 14173-14181.

14. Li Y, Yu N, Du L, Shi W, Yu H, et al. (2020) Transplacental transfer of per- and polyfluoroalkyl substances identified in paired maternal and cord sera using suspect and nontarget screening. Environ Sci Technol 54: 3407-3416.

15. Morgan D, Tsai SC (2015) Sleep and the endocrine system. Crit Care Clin 31: 403-418.

16. Sanderson JT (2006) The steroid hormone biosynthesis pathway as a target for endocrine-disrupting chemicals. Toxicol Sci 94: 3-21.

17. Chen G, Jin Y, Wu Y, Liu L, Fu Z (2015) Exposure of male mice to two kinds of organophosphate flame retardants (OPFRs) induced oxidative stress and endocrine disruption. Environ Toxicol Pharmacol 40: 310-318.

18. Chianese R, Viggiano A, Urbanek K, Cappetta D, Troisi J, et al. (2018) Chronic exposure to low dose of bisphenol A impacts on the first round of spermatogenesis via SIRT1 modulation. Sci Rep 8: 2961.

19. Santoro A, Chianese R, Troisi J, Richards S, Nori SL, et al. (2019) Neuro-toxic and reproductive effects of BPA. Curr Neuropharmacol 17: 1109-1132.

20. Cescon M, Chianese R, Tavares RS (2020) Environmental impact on male (in)fertility via epigenetic route. $J$ Clin Med 9: 2520.

21. Sharpe RM, Irvine DS (2004) How strong is the evidence of a link between environmental chemicals and adverse effects on human reproductive health? BMJ 328: 447-451.

22. Kaise A, Ohta K, Fujii S, Oda A, Goto T, et al. (2018) Novel androgen receptor full antagonists: Design, synthesis, and a docking study of glycerol and aminoglycerol derivatives that contain p-carborane cages. Bioorg Med Chem 26: 3805-3811.

23. Yuan X, Shen J, Zhang X, Tu W, Fu Z, et al. (2020)
Imidacloprid disrupts the endocrine system by interacting with androgen receptor in male mice. Sci Total Environ 708: 135163.

24. Zhu Q, Li H, Wen Z, Wang Y, Li X, et al. (2020) Perfluoroalkyl substances cause Leydig cell dysfunction as endocrine disruptors. Chemosphere 253: 126764.

25. Kjeldsen LS, Bonefeld-Jørgensen EC (2013) Perfluorinated compounds affect the function of sex hormone receptors. Environ Sci Pollut Res Int 20: 8031-8044.

26. Crawford NM, Fenton SE, Strynar M, Hines EP, Pritchard DA, et al. (2017) Effects of perfluorinated chemicals on thyroid function, markers of ovarian reserve, and natural fertility. Reprod Toxicol 69: 53-59.

27. Fenton SE, Reiner JL, Nakayama SF, Delinsky AD, Stanko JP, et al. (2009) Analysis of PFOA in dosed CD-1 mice. Part 2. Disposition of PFOA in tissues and fluids from pregnant and lactating mice and their pups. Reprod Toxicol 27: 365-372.

28. Lau C, Thibodeaux JR, Hanson RG, Narotsky MG, Rogers JM, et al. (2006) Effects of perfluorooctanoic acid exposure during pregnancy in the mouse. Toxicol Sci 90 : $510-518$.

29. Gützkow KB, Haug LS, Thomsen C, Sabaredzovic A, Becher G, et al. (2012) Placental transfer of perfluorinated compounds is selective - a Norwegian mother and child sub-cohort study. Int J Hyg Environ Health 215: 216-219.

30. Fenton SE (2006) Endocrine-disrupting compounds and mammary gland development: early exposure and later life consequences. Endocrinology 147(6 Suppl): S18-S24.

31. White SS, Fenton SE, Hines EP (2011) Endocrine disrupting properties of perfluorooctanoic acid. J Steroid Biochem Mol Biol 127: 16-26.

32. Barlow NJ, Phillips SL, Wallace DG, Sar M, Gaido KW, et al. (2003) Quantitative changes in gene expression in fetal rat testes following exposure to di(n-butyl) phthalate. Toxicol Sci 73: 431-441.

33. Tu W, Martínez R, Navarro-Martin L, Kostyniuk DJ, Hum C, et al. (2019) Bioconcentration and metabolic effects of emerging PFOS alternatives in developing zebrafish. Environ Sci Technol 53: 13427-13439.

34. Wang C, Zhao Y, Jin Y (2020) The emerging PFOS alternative OBS exposure induced gut microbiota dysbiosis and hepatic metabolism disorder in adult zebrafish. Comp Biochem Physiol C Toxicol Pharmacol 230: 108703.

35. Wang C, Zhang Y, Deng M, Wang X, Tu W, et al. (2019) Bioaccumulation in the gut and liver causes gut barrier dysfunction and hepatic metabolism disorder in mice after exposure to low doses of OBS. Environ Int 129: 279-290.

36. Xing J, Wang G, Zhao J, Wang E, Yin B, et al. (2016) Toxicity assessment of perfluorooctane sulfonate using acute and subchronic male C57BL/6J mouse models. Environ pollut 210: 388-396.

37. Duan P, Hu C, Butler HJ, Quan C, Chen W, et al. (2016) Effects of 4-nonylphenol on spermatogenesis and induction of testicular apoptosis through oxidative stress-related pathways. Reprod Toxicol 62: 27-38.

38. Johnsen SG (1970) Testicular biopsy score count-a 
method for registration of spermatogenesis in human testes: normal values and results in 335 hypogonadal males. Hormones 1: 2-25.

39. Trivedi PP, Kushwaha S, Tripathi DN, Jena GB (2010) Evaluation of male germ cell toxicity in rats: correlation between sperm head morphology and sperm comet assay. Mutat Res 703: 115-121.

40. Zeng Q, Yi H, Huang L, An Q, Wang H (2018) Reduced testosterone and Ddx3y expression caused by long-term exposure to arsenic and its effect on spermatogenesis in mice. Environ Toxicol Pharmacol 63: 84-91.

41. Jin C, Zhang R, Fu Z, Jin Y (2019) Maternal exposure to imazalil disrupts the endocrine system in $\mathrm{F} 1$ generation mice. Mol Cell Endocrinol 486: 105-112.

42. Jin C, Yuan X, Wang C, Fu Z, Jin Y (2021) Maternal exposure to imazalil disrupts intestinal barrier and bile acids enterohepatic circulation tightly related IL-22 expression in F0, F1 and F2 generations of mice. J Hazard Mater 403: 123668.

43. Livak KJ, Schmittgen TD (2001) Analysis of relative gene expression data using realtime quantitative PCR and the 2(-Delta Delta C(T)) method. Methods 25: 402-408.

44. Ni Y, Zhao Y, Ma L, Wang Z, Ni L, et al. (2021) Pharmacological activation of REV-ERB $\alpha$ improves nonalcoholic steatohepatitis by regulating intestinal permeability. Metabolism 114: 154409.

45. Jin Y, Wang L, Ruan M, Liu J, Yang Y, et al. (2011) Cypermethrin exposure during puberty induces oxidative stress and endocrine disruption in male mice. Chemosphere 84: 124-130.

46. Xia J, Jin C, Pan Z, Sun L, Fu Z, et al. (2018) Chronic exposure to low concentrations of lead induces metabolic disorder and dysbiosis of the gut microbiota in mice. Sci Total Environ 631-632: 439-448.

47. Chen J, Zheng L, Tian L, Wang N, Lei L, et al. (2018) Chronic PFOS exposure disrupts thyroid structure and function in zebrafish. Bull Environ Contam Toxicol 101: 75-79.

48. Fromme H, Mosch C, Morovitz M, Alba-Alejandre I, Boehmer S, et al. (2010) Pre- and postnatal exposure to perfluorinated compounds (PFCs). Environ Sci Technol 44: 7123-7129.

49. Zhang H, He J, Li N, Gao N, Du Q, et al. (2019) Lipid accumulation responses in the liver of Rana nigromaculata induced by perfluorooctanoic acid (PFOA). Ecotoxicol Environ Saf 167: 29-35.

50. Shi Z, Zhang H, Liu Y, Xu M, Dai J (2007) Alterations in gene expression and testosterone synthesis in the testes of male rats exposed to perfluorododecanoic acid. Toxicol Sci 98: 206-215.

51. Azhar S, Reaven E (2002) Scavenger receptor class BI and selective cholesteryl ester uptake: partners in the regulation of steroidogenesis. Mol Cell Endocrinol 195: 1-26.

52. Clark BJ, Soo SC, Caron KM, Ikeda Y, Parker KL, et al. (1995) Hormonal and developmental regulation of the steroidogenic acute regulatory protein. Mol Endocrinol 9: $1346-1355$.
53. Stocco DM, Clark BJ (1996) Regulation of the acute production of steroids in steroidogenic cells. Endocr Rev 17: 221-244.

54. Dziendzikowska K, Krawczyńska A, Oczkowski M, Królikowski T, Brzóska K, et al. (2016) Progressive effects of silver nanoparticles on hormonal regulation of reproduction in male rats. Toxicol Appl Pharmacol 313: 35-46.

55. Deng M, Wu Y, Xu C, Jin Y, He X, et al. (2018) Multiple approaches to assess the effects of F-53B, a Chinese PFOS alternative, on thyroid endocrine disruption at environmentally relevant concentrations. Sci Total Environ 624: 215-224.

56. Shi G, Cui Q, Pan Y, Sheng N, Sun S, et al. (2017) 6: 2 Chlorinated polyfluorinated ether sulfonate, a PFOS alternative, induces embryotoxicity and disrupts cardiac development in zebrafish embryos. Aquat Toxicol 185: 67-75.

57. Shi G, Guo H, Sheng N, Cui Q, Pan Y, et al. (2018) Twogenerational reproductive toxicity assessment of 6: 2 chlorinated polyfluorinated ether sulfonate (F-53B, a novel alternative to perfluorooctane sulfonate) in zebrafish. Environ Pollut 243: 1517-1527.

58. Singh S, Singh SK (2019) Prepubertal exposure to perfluorononanoic acid interferes with spermatogenesis and steroidogenesis in male mice. Ecotoxicol Environ Saf 170: 590-599.

59. Singh S, Singh SK (2019) Chronic exposure to perfluorononanoic acid impairs spermatogenesis, steroidogenesis and fertility in male mice. J Appl Toxicol 39: 420-431.

60. Lai KP, Lee JC, Wan HT, Li JW, Wong AY, et al. (2017) Effects of in utero PFOS exposure on transcriptome, lipidome, and function of mouse testis. Environ Sci Technol 51: 8782-8794.

61. Zhang H, Lu H, Chen P, Chen X, Sun C, et al. (2020) Effects of gestational perfluorooctane sulfonate exposure on the developments of fetal and adult Leydig cells in F1 males. Environ Pollut 262: 114241.

62. Cheng CY, Mruk DD (2012) The blood-testis barrier and its implications for male contraception. Pharmacol Rev 64: 16-64.

63. Mao B, Mruk D, Lian Q, Ge R, Li C, et al. (2018) Mechanistic insights into PFOS-Mediated sertoli cell injury. Trends Mol Med 24: 781-793.

64. Jin H, Ma T, Sha X, Liu Z, Zhou Y, et al. (2021) Polystyrene microplastics induced male reproductive toxicity in mice. J Hazard Mater 401: 123430.

65. Li MWM, Mruk DD, Lee WM, Cheng CY (2010) Connexin 43 is critical to maintain the homeostasis of the blood-testis barrier via its effects on tight junction reassembly. Proc Natl Acad Sci U S A 107: 17998-18003.

66. Svechnikov K, Izzo G, Landreh L, Weisser J, Söder O (2010) Endocrine disruptors and Leydig cell function. $J$ Biomed Biotechnol 2010: 684504.

67. Sajadi E, Dadras S, Bayat M, Abdi S, Nazarian H, et al. (2019) Impaired spermatogenesis associated with changes in spatial arrangement of sertoli and spermatogonial cells following induced diabetes. J Cell Biochem 120: 17312- 
17325.

68. Simpson E, Rubin G, Clyne C, Robertson K, O’Donnell L, et al. (2000) The role of local estrogen biosynthesis in males and females. Trends Endocrinol Metab 11: 184188.

69. Rochira V, Carani C (2009) Aromatase deficiency in men: a clinical perspective. Nat Rev Endocrinol 5: 559-568.

70. Jones ME, Boon WC, Mclnnes K, Maffei L, Carani C, et al. (2007) Recognizing rare disorders: aromatase deficiency. Nat Clin Pract Endocrinol Metab 3: 414-421.

71. Rochira V, Balestrieri A, Madeo B, Spaggiari A, Carani C (2002) Congenital estrogen deficiency in men: a new syndrome with different phenotypes; clinical and therapeutic implications in men. Mol Cell Endocrinol 193: 19-28.

72. Carani C, Qin K, Simoni M, Faustini-Fustini M, Serpente
S, et al. (1997) Effect of testosterone and estradiol in a man with aromatase deficiency. $N$ Engl J Med 337: 91-95.

73. Morishima A, Grumbach MM, Simpson ER, Fisher C, Qin K (1995) Aromatase deficiency in male and female siblings caused by a novel mutation and the physiological role of estrogens. J Clin Endocrinol Metab 80: 36893698.

74. Czajka-Oraniec I, Simpson ER (2010) Aromatase research and its clinical significance. Endokrynol Pol 61: 126-134.

75. Bouchoucha N, Samara-Boustani D, Pandey AV, BonyTrifunovic H, Hofer G, et al. (2014) Characterization of a novel CYP19A1 (aromatase) R192H mutation causing virilization of a 46, XX newborn, undervirilization of the 46, XY brother, but no virilization of the mother during pregnancies. Mol Cell Endocrinol 390: 8-17. 